\title{
Caracterisation Agro-Morphologique Des Accessions D’arachide (Arachis Hypogaea L.) Pour La Teneur En Huile
}

\author{
Alleidi Issa \\ Université Abdou Moumouni de Niamey, Niger \\ Hamidou Falalou \\ ICRISAT/Sadoré/Niamey/Niger \\ Inoussa Manan Maârouhi \\ Bakasso Yacoubou \\ Université Abdou Moumouni de Niamey, Niger \\ Zongo Jean Didier
}

Université de OuagaI Pr Joseph KI-ZERBO, Burkina Faso

doi: 10.19044/esj.2016.v12n15p337 URL:http://dx.doi.org/10.19044/esj.2016.v12n15p337

\begin{abstract}
In Niger, groundnut [Arachis hypogaea (L.)] is a leguminous crop cultivated in large area. Its seeds are used by oil industries. Groundnut oil is well appreciated by consumers notably in rural areas. The aim of this study was to select the accessions and taxa with high oil content and identify the oil content related traits. Thus, 20 accessions (9 local and 11 improved) belonging to 5 taxa types (FST, VUL, PRU, HYR, and HYB) were planted in randomized block design with three replications. Fifteen traits were investigated in field while oil content was quantified in laboratory. An analysis of variance was performed on all measured traits. Discriminant factorial analysis (AFD) was performed on taxon types, correlation between oil content and other traits was determined. Analysis revealed high and significant correlation between oil content and seed size. In the study, groundnut accessions ICG 8352 and ICG 9991 (oil content 52.8\%) and taxon Fastigiata (oil content 48.52\%) recorded highest oil content. Seed size and taxon type in groundnut could be criteria to select genotypes for oil industries.
\end{abstract}

Keywords: Groundnut, Accession, Taxon, Oil content, Pod yield, Legume.

\section{Résumé}

L'arachide [Arachis hypogaea (L.)] est une plante oléagineuse cultivée au Niger sur une large superficie. Sa graine est très utilisée dans les 
industries d'huilerie à cause de sa richesse en huile qui est très appréciée par les consommateurs notamment le monde rural. La présente étude a pour objectif d'identifier les meilleures accessions et types taxonomiques riches en huile et déterminer les caractères corrélés à cette teneur en huile. Ainsi, les graines de 20 accessions ( 9 génotypes locaux et 11 améliorés) de 5 types taxonomiques (FST, VUL, PRU, HYR et HYB) ont été semées au champ selon un dispositif en blocs de Fisher randomisés avec 3 répétitions. Quinze caractères agromorphologiques ont été investigués au champ ou au laboratoire. Une analyse de variance a été réalisée pour l'ensemble des caractères étudiés tant pour les accessions que les types taxonomiques. Afin de spécifier les taxons et déterminer le niveau de corrélation entre la teneur en huile et les autres caractères, une Analyse Factorielle Discriminante (AFD) et une matrice de corrélation ont été effectuées. Les études de corrélation ont révélé que le poids moyen d'une graine est positivement corrélé à la teneur en huile. Les accessions ICG 8352 et ICG 9991 (teneur huile 52,8\%) ainsi que le taxon Fastigiata (teneur en huile 48,52\%) se sont révélées meilleures accessions dans cette étude. Le poids moyen d'une graine et le type taxonomique seraient de bons critères pour identifier les génotypes d'arachide d'industrie d'huilerie.

Mots clefs : Arachide, Accession, Taxon, Teneur en huile, Rendement en gousses, Oleagineux

\section{Introduction}

L'arachide, (Arachis hypogaea L.), est une espèce tétraploïde (Singh et al., 1998; Herselman, 2003). Elle a une garniture chromosomique de $2 n=40$. Cette plante est une autogame. Néanmoins son taux d'allogamie est de 0,2 \% pour le type Virginia. Par contre, ce taux est de 6,6 \% pour ceux de Spanish (Sauger, 1949) et Valencia (Bouffil, 1951) dans les zones où l'activité des abeilles est intense (Nigam et al., 1983). Elle est originaire, d'Amérique du Sud (Hammons, 1973 et KhalfaouI, 1988). Elle est l'une des plantes oléagineuses la plus cultivée en Afrique Occidentale. Cette plante comprend plus de soixante espèces annuelles et pérennes; dont l'espèce hypogaea a elle seule, présente deux sous-espèces à savoir: Arachis hypogaea hypogaea et Arachis hypogaea fastigiata (Gregory et al., 1976; et Krapovickas, 1969). Chacune de ces sous-espèces, compte au moins deux variétés: les variétés hypogaea et hirsuta appartiennent à la sous espèce hypogaea et les variétés fastigiata, vulgaris, aequatoriana et peruviana appartiennent à la sousespèce fastigiata. Les types, les plus cultivés sont: Virginia bunch et runne; Valencia peruvian, Spanish et Peruvian hirsuta, Eaquatorian. L'arachide est une légumineuse largement produite dans le monde pour sa forte teneur en huile (Celia et al., 2005). Les graines sont couvertes d’un tégument séminal, 
rose ou saumon. Parfois, ce tégument présente plusieurs couleurs très variées en allant du blanc au marron (IBPGR et al., 1992). L'amande est composée de deux cotylédons gorgés de matières grasses et d'un embryon facilement distinguable. Cette amande est très utilisée dans les industries d'huileries à cause de sa richesse en huile. L'arachide est une espèce oléagineuse; elle est l'une des rares légumineuses à fructification souterraine à être utilisée dans le monde (Mayeux, 1999). Elle est riche en huile (44 et $56 \%$ ), en protéines (22 à $30 \%$ ), en potassium, en phosphate et en magnésium (Conkertone et al., 1989)). Sa teneur en huile, son adaptabilité, sa capacité de restauration de la fertilité des sols et son utilisation dans l'alimentation de bétail font, d'elle une excellente plante oléagineuse au Niger. La teneur en huile d'arachide est variable selon les types taxonomiques. En effet, la sous espèce Arachis hypogaea hypogaea a une teneur en huile qui varie entre 36 et $47 \%$ d'huile. Tandis que, celle de la sous espèce Arachis hypogaea fastigiata varie entre 45 et $50 \%$ (Celia et al., 2005).

Cette étude a pour objectif d'évaluer les accessions d'arachide afin d‘identifier la meilleure accession riche en huile et de déterminer le caractère qui est lié à la teneur en huile.

\section{Matériel et Méthodes Matériel végétal:}

Le matériel utilisé est constitué de 20 accessions d'arachide locales (9) et améliorées (11) de la banque de gènes de l'ICRISAT Sadoré /Say/Niger). Cinq types taxonomiques (fastigiata vulgaris Spanish (VUL), fastigiata fastigiata Valancia (FST), fastigiata peruviana Valencia peruviana (PRU) hypogaea runner Virginia runner (HYR), hypogaea bunch Virginia bunch $(H Y B)$ ) et diverses origines (Mali, Sénégal etc.) ont été sélectionnés pour cette étude (Tableau 1).

Tableau 1: Taxon, origine, donneur et iso donneur du matériel végétal utilisé

\begin{tabular}{|c|c|c|c|c|c|c|c|c|c|c|c|c|c|}
\hline Code & Génotypes & Taxon & BST & Sources & Donneur & $\begin{array}{l}\text { Iso- } \\
\text { donneur }\end{array}$ & Code & Génotypes & Taxon & BST & Sources & Donneur & $\begin{array}{l}\begin{array}{l}\text { Iso- } \\
\text { donneur }\end{array} \\
\end{array}$ \\
\hline A1 & ICG 10005 & PRU & LR & 5PER & IAC & IND & A11 & ICG 8550 & FST & LR & 9MDG & IAC & IND \\
\hline A2 & ICG 8352 & HYB & $\mathrm{BL}$ & 9TWN & IAC & IND & A12 & ICG 8594 & PRU & LR & 9ZAR & IAC & IND \\
\hline A3 & ICG 8385 & HYB & $\mathrm{BL}$ & 9USA & IAC & IND & A13 & ICG 8606 & $\begin{array}{l}\text { FST } \\
\end{array}$ & $\mathrm{BL}$ & 9WZE & IAC & IND \\
\hline A4 & ICG 8398 & HYB & $\mathrm{BL}$ & 9USA & IAC & IND & A14 & ICG 8724 & HYB & BL & 9MLI & IAC & IND \\
\hline A5 & ICG 8429 & HYR & LR & 2MWI & IAC & IND & A15 & ICG 8746 & HYR & $\mathrm{BL}$ & 9SEN & IAC & IND \\
\hline A6 & ICG 8431 & VUL & LR & 2MWI & IAC & IND & A16 & ICG 8781 & HYR & LR & 2ZMB & IAC & IND \\
\hline A7 & ICG 8439 & HYR & LR & 5MWI & IAC & IND & A17 & ICG 8848 & FST & $\mathrm{BL}$ & 9ZWE & IAC & IND \\
\hline A8 & ICG 8443 & VUL & LR & 9ZAF & IAC & IND & A18 & ICG 8863 & FST & $\mathrm{BL}$ & 9ZWE & IAC & IND \\
\hline A9 & ICG 8454 & VUL & $\mathrm{BL}$ & 9ZWE & IAC & IND & A19 & ICG 9989 & PRU & LR & 9PER & IAC & IND \\
\hline A10 & ICG 8460 & VUL & LR & 9ZWE & IAC & IND & A20 & ICG 9991 & PRU & LR & 5PER & IAC & IND \\
\hline
\end{tabular}

A: Accession; Génotypes: ICG, Icrisat Centre Groundnut (Arachide);Taxonomique: VUL, Vulgaris; FST, Fastigiata; PRU, Perivian; HYR, Hirsuta; HYB, Bunch ; BST: BL, breeder's line; LR, Landrace;

Sources: MLI, Mali; MWI, Malawi; MDG, Madagascar; PER, Pérou; SEN, Sénégal; TWN, Taiwan; USA, Etat Unies d’Amérique; ZAF, Afrique du Sud; ZAR, Zaïre; ZWE, Zimbabwe; ZMB, Zombie; Donneurs: IAC, Collection international d’Arachide ; Iso donneurs: IND, Inde. 


\section{Méthodes:}

\section{Site d'expérimentation}

L’essai a été conduit au sein de la station de recherche de l'ICRISAT Sadoré/ Say/ Niger. Le centre s’étend sur 500 ha et est situé à 40 km au SudEst de Niamey (capitale du Niger), à $22 \mathrm{~km}$ Nord-Ouest de Say et $7 \mathrm{~km}$ à l'Ouest du fleuve Niger. Il se situe entre latitude $13^{\circ} 15^{\prime} \mathrm{N}$ et longitude 2॰18’E.

Le dispositif utilisé, était un bloc de Fisher randomisé. Les graines des 20 accessions ont été mises en culture selon le dispositif de blocs de Fisher Randomisés avec trois (3) répétitions. Chaque répétition est constituée de 20 parcelles élémentaires de dimension de $4800 \mathrm{~cm}^{2}(60 \mathrm{~cm}$ x $80 \mathrm{~cm})$. Les écartements entre les répétitions d'une part, et d'autre part entre les parcelles est de $1 \mathrm{~m}$. Chaque parcelle dispose de deux lignes de semis, écartées de 60 $\mathrm{cm}$. Chaque ligne comportait cinq (5) poquets écartés de $20 \mathrm{~cm}$.

Avant le semis, une fumure de fond a été appliquée. Cette application ou l' épandage est composé d'un mélange de fumier et de l'engrais NPK (14-23-14). Après cet épandage de fumure de fond, un labour a été effectué. Le semis a été effectué, après une pluie de 18,2 mm. Trois graines ont été semées par poquet.

\section{Collectes des données}

La culture a fait l'objet d'observations depuis le stade semis jusqu'à la date de récolte. La collecte de données (12 caractères) a été faite selon les indicateurs de la liste des descripteurs de l'arachide (IBPGR et al., 1992). Les 3 autres caractères ont été déterminés de la façon suivante: -la surface foliaire (SF) a été mesurée à l'aide d'un appareil Area Meter LI 3100; sa détermination a consisté à prélever la troisième feuille de la tige principale de trois plantes par accession dans une répétition, puis la scanner avec cet appareil;- l'indice de surface foliaire (LAI) a été déterminé au $70^{\text {ème }} \mathrm{JAS}$ avec l’appareil LAI -2000PCA.

\section{Extraction d'huile}

La teneur en huile (TEH) a été quantifiée par l'extraction d'huile. L'huile a été extraite à partir de l'hexane avec l'appareil de Soxhlet pendant 3 heures. Les étapes de cette extraction d'huile sont les suivantes:

- $\quad$ Oter la pellicule des graines (tégument séminal).

- Broyer les graines.

- $\quad$ Peser 5 g de broyat par accessions.

- $\quad$ Emballer avec papier filtre et le mettre dans le soxhlet.

- Mettre en place la plaque chauffante à $70^{\circ} \mathrm{C}$, le système de refroidissement et la pierre ponce dans le ballon à col rodé (29/32). 
- $\quad$ Peser le ballon contenant la pierre ponce (P0).

- $\quad$ Faire couler l'hexane (200 ml) dans le soxhlet contenant l'échantillon.

- $\quad$ Mettre en marche la plaque chauffante et le système de refroidissement pendant une durée de 3 heures dont le nombre de siphonageS varie de 12 à 14 .

- $\quad$ Evaporer l’hexane au rotavapor à la température d’ébullition du solvant $\left(61^{\circ} \mathrm{C}\right)$.

- $\quad$ Mettre le ballon contenant l'huile à l'étuve à $100^{\circ} \mathrm{C}$.

- $\quad$ Effectuer une série de pesées (3 fois) à chaque 40 mn afin d’obtenir un poids constant de l'échantillon.

- Calculer la teneur en huile selon la formule suivante: TEH(\%)= $100 *(\mathrm{P} 1-\mathrm{P} 0) / \mathrm{E}$, avec $\mathrm{P} 0=$ poids du ballon vide contenant la pierre et $\mathrm{P} 1=$ poids du ballon contenant l'huile plus la pierre ponce, $\mathrm{E}=$ prise d'essai (5 g).

\begin{tabular}{|l|l|l|l|}
\hline Paramètres & Abréviations & Paramètres & Abréviations \\
\hline 1. Taux de germination & TAG (\%) & 8. Durée du cycle & DC (jours \\
\hline 2. Levée & T (\%) & $\begin{array}{l}\text { 9. Nombre de gousses par } \\
\text { plante }\end{array}$ & NGP \\
\hline 3. Début de floraison & DFL (jours) & $\begin{array}{l}\text { 10. Rendement en gousses } \\
\text { par plante }\end{array}$ & $\begin{array}{l}\text { RGP } \\
\text { (g/plante) }\end{array}$ \\
\hline $\begin{array}{l}\text { 4. Cinquante pour cent de } \\
\text { floraison }\end{array}$ & $\mathbf{5 0 ~ \% ~ F ~ ( j o u r s ) ~}$ & $\begin{array}{l}11 . \text { Nombre de réticulations } \\
\text { par gousse }\end{array}$ & NRG \\
\hline 5. Surface foliaire & SF $\left.\mathbf{( c m}^{\mathbf{2}}\right)$ & $\begin{array}{l}\text { 12. Nombre maximum de } \\
\text { graines par gousse }\end{array}$ & Nmg/G \\
\hline $\begin{array}{l}\text { 6. Hauteur de la tige principale } \\
\text { de la plante }\end{array}$ & HPL (cm) & $\begin{array}{l}\text { 13. Poids moyen d'une } \\
\text { graine }\end{array}$ & PMg (g) \\
\hline 7. Indice de surface foliaire & LAI & $\begin{array}{l}\text { 14. Rendement en graines } \\
\text { par plante }\end{array}$ & $\begin{array}{l}\text { RgP } \\
\text { (g/plante }\end{array}$ \\
\hline 15. Teneur en huile & TEH (\%) & \\
\hline
\end{tabular}

Tableau 2: Les 15 variables quantitatives pour évaluer les 20 accessions d'arachide

\section{Analyse des données}

Les données collectées (données brutes) ont été traitées par le logiciel Excel pour en faire des données statistiques. Ce logiciel a été aussi utilisé pour voir le niveau de corrélation entre la teneur en huile et les autres caractères étudiés.

Les analyses de variance des différents paramètres ont été faites par le logiciel XLSTAT version 7.1 avec un intervalle de confiance de $95 \%$, soit un risque alpha de $5 \%$. Ce logiciel a servi pour l'AFD (Analyse Factorielle Discriminant) pour tester la variabilité morphologique des différents types taxonomiques. Le regroupement des accessions a été fait suivant leurs performances et en fonction des objectifs attendus, trois types de test ont été retenus à savoir le test de ppds (plus petite différence significative protégée), le test de Duncan et le test de Tukey. 


\section{Résultats et Discussion \\ Paramètres bioclimatiques}

Le développement des plantes, s’est effectué sous les paramètres climatiques de la station de recherche de l'ICRISAT/Sadoré/Say/Niger. La température moyenne journalière et la radiation solaire évoluent ensemble. En effet, plus la radiation solaire est élevée, plus la température est importante. Ces deux facteurs augmentent du 26 Août au 5 Septembre et se stabilisent relativement vers la période froide. Quant à l'humidité relative, elle était plus importante pendant la saison pluvieuse. Elle diminue progressivement vers la fin de cette saison et ensuite croît faiblement à nouveau à partir du début de la période froide. Cette humidité relative évolue de façon aléatoire avec la température. Ces deux paramètres avaient presque la même valeur à la date du 04 Novembre. Cette humidité relative évolue en antagonisme avec la vitesse du vent. C'est-à-dire: plus, elle augmente, plus la vitesse du vent diminue. Cette vitesse du vent augmente l'évaporation du bac (figure 1). Les sommes de ces facteurs climatiques durant l'essai sont: Température (2 939,1 $\left.{ }^{\circ} \mathrm{C}\right)$; pluviométrie moyenne $(54,8 \mathrm{~mm} / \mathrm{j})$; humidité relative (5318\%); évaporation du bac $(648,3 \mathrm{~mm} / \mathrm{j})$; radiation solaire (2 $182,3 \mathrm{MJ} / \mathrm{m}^{2}$ ). Quant à la vitesse du vent, elle a été estimée à 310,4 m/s. La figure 2, montre l'évolution de la température journalière du stade remplissage des gousses à la récolte. Cette température a fluctué entre $27,6^{\circ}$ $\mathrm{C}$ et $30,7^{\circ} \mathrm{C}$ avec une somme de température journalière $\left(229,3^{\circ} \mathrm{C}\right)$.

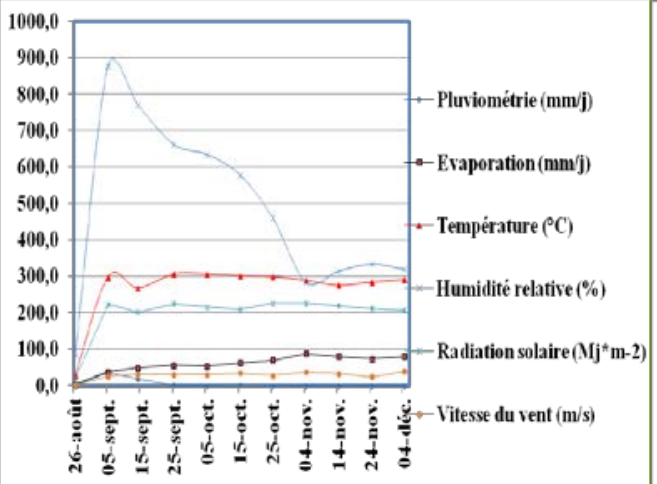

Figure 1: Evolution des facteurs climatiques durant l'expérimentation

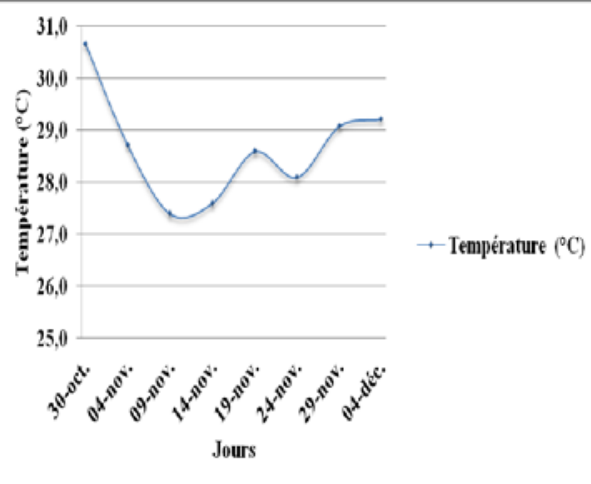

Figure 2: Evolution de la température journalière durant la période de remplissage des gousses à la récolte au champ

\section{Caractères quantitatifs}

\section{Degré de signification des paramètres étudiés}

Parmi, les quinze caractères quantitatifs; cinq caractères quantitatifs pré-récolte (caractères mesurés avant la récolte) montrent des différences significatives entre les accessions évaluées. Par ailleurs, l'analyse de variance n'a pas porté sur les caractères tels que le début de floraison (DFL), l'indice de surface foliaire (LAI), le nombre de réticulations par gousse 
(NRG), le nombre maximum des graines par gousse $(\mathrm{Nmg} / \mathrm{G})$ et le poids moyen d'une graine (PMg).

Quant aux caractères quantitatifs post-récolte, le nombre de gousses par plante (NGP), le rendement en gousses par plante (RGP) et le rendement en graines par plante (RgP) ne montrent aucune différence significative. En outre, le taux de germination (TAG) varie entre 22,67\% (A15) et $100 \%$ (A13, A19 et A20).

L’ANOVA montre une différence très hautement significative $\left(\mathrm{F}=6,04^{* * *}\right)$ entre les accessions étudiées pour ce taux de germination, avec un coefficient de détermination $\left(\mathrm{R}^{2}=74,2 \%\right)$ et un coefficient de corrélation $(\mathrm{R}=0,86)$. Le test de Tukey regroupe les accessions en quatre sous groupes.

Le taux de levée varie entre 26,67 (A15) et $100 \%$ (A20). Ce pourcentage est inférieur à celui de la germination pour toutes les accessions, exceptées les accessions A8, A9, A7, A11, A14, A15, A17, A18 et A20 où leurs taux de levée sont voisins de leurs taux de germination.

Quant à la date de floraison, les accessions A13 et A5 fleurissent entre 19 et 25 JAS respectivement (Tableau 4). Alors que, la date de cinquante pour cent de floraison (50 \% F) varie entre 23 JAS (A13) et 35 JAS (A15); une différence très hautement significative $(\mathrm{F}=7,07 * * *)$, a été détectée. Les coefficients de détermination et de corrélation sont respectivement $\mathrm{R}^{2}=66,2 \%$ et $\mathrm{R}=0,87$. Pour le test de ppds, sept sous groupes ont été distingués.

La surface foliaire des accessions d'arachide varie entre $13,951 \mathrm{~cm}^{2}$ (A14) et 34,73 $\mathrm{cm}^{2}$ (A17) avec une moyenne de 22,30 $\pm 8,01 \mathrm{~cm}^{2}$. La différence est significative $\left(\mathrm{F}=2,00^{*}\right)$ entre les accessions. Les coefficients de corrélation et de détermination sont $\mathrm{R}=0,69$ et $\mathrm{R}^{2}=48,8 \%$. Trois sous groupes ont été identifiés par le test de Duncan.

La hauteur de la plante (HPL) varie entre $7 \mathrm{~cm}$ (A15) et 24,16 cm (A13) avec une moyenne de 13,56 \pm 6,5 cm. L’ANOVA montre une différence très significative $\left(\mathrm{F}=2,66^{* *}\right)$ entre les accessions d'arachide. Les coefficients de corrélation et de détermination sont $\mathrm{R}=0,74$ et $\mathrm{R}^{2}=55,8 \%$ respectivement. Selon le test de Duncan, les accessions ont été classées en quatre sous groupes (Tableau 4).

La durée du cycle s’étale de 80 jours (A8) à 98,33 jours (A1) avec une moyenne de 90,75 $\pm 6,70$ jours. L'analyse de variance montre une différence hautement significative $\left(\mathrm{F}=2,95^{* *}\right)$. Les coefficients de corrélation et de détermination sont respectivement $\mathrm{R}=0,76$ et $\mathrm{R}^{2}=58,4 \%$. Le test de ppds regroupe les accessions en six sous groupes.

Le nombre de gousses par plante (NGP), se fluctue entre 18,60 (A20) et 71,33 (A7) avec une moyenne de 36, $65 \pm 21,17$ gousses par plante. L'ANOVA ne montre aucune différence significative $\left(F=1,20^{\text {ns }}\right)$. Le test de ppds a permis de classer les accessions en trois sous entités. 
Quant au rendement en gousses par plante (RGP), il varie entre 9,20 g/plante (A3) et 57,74 g/plante (A7) avec une moyenne de 21,61 \pm 21,70 g/plante. L’ANOVA ne montre aucune différence significative entre les moyennes $\left(\mathrm{F}=0,11^{\mathrm{ns}}\right)$.

Le rendement en graines par plante (RgP), varie entre 1,11 g/plante (A19) et 30,83 g/plante (A7) avec une moyenne de 9,03 \pm 13,43 g/plante. Le test de ppds reclasse les accessions en trois groupes.

La teneur en huile des accessions d'arachide varie entre 36,30\% (A8) et 52,80 \% (A20, A2) avec une moyenne de 44,92 $12,34 \%$. L’analyse de variance ne montre aucune différence significative $\left(F=0,29^{\text {ns }}\right)$ entre les accessions d'arachide au risque alpha égale à $5 \%$. L'analyse de variance des caractères au sein des répétitions ne montre aucune différence significative pour le taux de germination, la date de 50 \% de floraison, la durée du cycle, le nombre de gousses par plante, le rendement en gousses par plante, le rendement en graines par plante, la teneur en huile. Tandis que, des différences significatives sont enregistrées pour la surface foliaire $\left(\mathrm{F}=4,22^{*}\right)$ et la hauteur de la plante $\left(\mathrm{F}=3,04^{*}\right)$ (Tableau 3).

Tableau 3: Performance de l'ensemble des 20 accessions d'arachide

\begin{tabular}{|c|c|c|c|c|c|c|c|c|c|c|}
\hline & caractères & $\begin{array}{l}\text { TAG } \\
\text { (J) }\end{array}$ & $\begin{array}{l}50 \mathrm{~F} \\
\%(\mathrm{~J})\end{array}$ & $\begin{array}{l}\text { SF } \\
\left(\mathrm{cm}^{2}\right)\end{array}$ & $\begin{array}{l}\text { HPL } \\
(\mathrm{cm})\end{array}$ & DC (J) & NGP & $\begin{array}{l}\text { RGP } \\
\text { (g/plante) }\end{array}$ & $\begin{array}{l}\text { RgP } \\
\text { (g/plante) }\end{array}$ & $\begin{array}{l}\text { TEH } \\
\text { (\%) }\end{array}$ \\
\hline Accessions & Fisher & $6,041^{* * *}$ & $7,079^{* * * *}$ & $2,005^{*}$ & $2,657^{* *}$ & $2,950^{* *}$ & $1,206^{\mathrm{ns}}$ & $0,119^{\text {ns }}$ & $1,496^{\text {ns }}$ & $0,296^{\text {ns }}$ \\
\hline Répétitions & Fisher & $0,0003^{\mathrm{ns}}$ & $0,131^{\mathrm{ns}}$ & $4,221^{*}$ & $3,034^{*}$ & $1,475^{\text {ns }}$ & $1,988^{\text {ns }}$ & $1,437^{\text {ns }}$ & $0,801^{\mathrm{ns}}$ & $0,135^{\mathrm{ns}}$ \\
\hline & Moyennes & 64,833 & 26,7 & 22,302 & 13,555 & 90,75 & 36,658 & 21,61 & 9,034 & 44,92 \\
\hline & CV (\%) & 44,2 & 3,7 & 35,9 & 44,7 & 7,4 & 57,8 & 10,05 & 14,87 & 27,5 \\
\hline & $\begin{array}{l}\text { Ecart } \\
\text { type }\end{array}$ & 28,671 & 3,233 & 8,011 & 6,057 & 6,691 & 21,171 & 21,61 & 13,433 & 12,343 \\
\hline & $\begin{array}{l}\text { Répétition } \\
1\end{array}$ & 64 & 26,4 & 18,474 & 11,885 & 89,25 & 30,835 & 15,61 & 5,921 & 43,28 \\
\hline & $\begin{array}{l}\text { Répétition } \\
2\end{array}$ & 65,5 & 26,8 & 22,254 & 12,625 & 90,25 & 35,365 & 22,728 & 10,509 & 46,56 \\
\hline & $\begin{array}{l}\text { Répétition } \\
3\end{array}$ & 64,900 & 26,900 & 25,374 & 16,057 & 92,75 & 43,775 & 26,744 & 10,672 & \\
\hline
\end{tabular}

***, très hautement significative, ${ }^{* *}$, hautement significative; ${ }^{*}$, significative; ${ }^{\text {ns }}$, non significative; $\mathbf{5 0} \% \mathbf{F}$, cinquante pourcent floraison; DC, durée du cycle; CV, coefficient de variation; HPL, hauteur de la plante; NGP, nombre de gousses par plante; RGP, rendement en gousses par plante; RgP, rendement en graine par plante; rep, répétition; SF, surface foliaire; TAG, taux de germination; TEH, teneur en huile.

\section{Teneur en huile}

La teneur en huile des accessions d'arachide varie entre 36,30\% (A8) et 52,80 \% (A20, A2) avec une moyenne de 44,92 $\pm 12,34 \%$. Elle est plus importante chez le type taxonomique FST (48,52 \%) que chez les types taxonomiques VUL (41,37 \%), PRU (43,85 \%), HYB (45,25 \%) et HYR (45,60\%). Ces résultats sont en accord avec ceux de Celia et al., (2005); selon lesquels, les accessions de type taxonomique FST sont plus riches en huile que les autres types taxonomiques. En particulier, les accessions A11 (49,20\%), A18 (49,30\%), A10 (50,90\%), A4 (51,0\%), A13 (51,30\%), A7 (51,60 \%), A2 (52,80 \%) et A20 (52,80 \%) sont les meilleures accessions de 
bonne teneur en huile (Tableau 4). Elles peuvent être considérées comme des accessions d'industrie (bonne teneur en huile). Alors que, les autres accessions seront considérées comme des accessions de «bouche». Cette importante teneur en huile du type taxonomique FST montre que, tout programme de création des variétés d'arachide de bonne teneur en huile doit tenir compte de la sous espèce fastigiata comme témoin. La matrice de corrélation, montre une corrélation significative entre la teneur en huile et le poids moyen d'une graine (Tableau 5). Cette corrélation est très faible. Elle pourrait être forte lorsque toutes les accessions appartenant au type taxonomique fastigiata fastigiata Valencia seront évaluées. Les huiles de quelques accessions sont présentées comme suit au niveau de figures 4 et 5 .

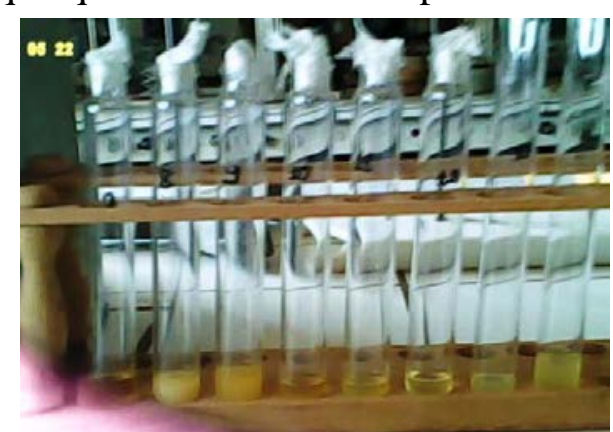

Figure 4: Aperçu des huiles de certaines Accessions

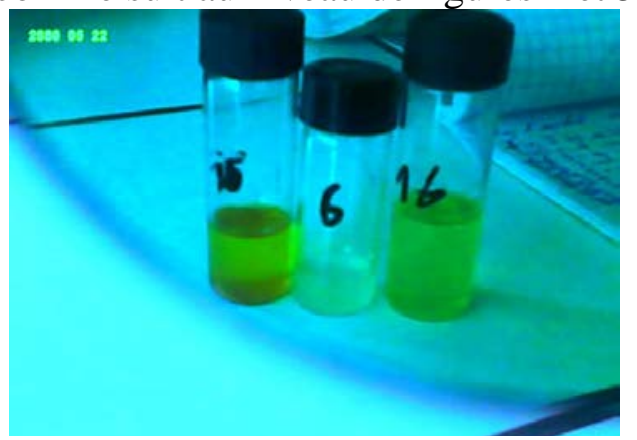

Figure 5: Huiles des accessions A3, A17, A8 respectivement de gauche à droite

\section{Performance des accessions d'arachide}

L'analyse de variance montre que la différence entre les moyennes des accessions, est significative pour le taux de germination, la date de $50 \%$ $\mathrm{F}$, la surface foliaire, la hauteur de la tige principale et la durée du cycle. Alors qu'aucune différence significative, n'est observée pour le nombre de gousses par plante, le rendement en gousses par plante, le rendement en graines par plante et la teneur en huile. Quatre caractères quantitatifs montrent une hétérogénéité au sein des 20 accessions évaluées. Le taux de germination ( $C V=44,20 \%)$, la surface foliaire $(C V=35,90 \%)$, la hauteur de la tige principale $(\mathrm{CV}=44,70 \%)$ et le nombre de gousses par plante ( $C V=36,65 \%)$ montrent que, les 20 accessions sont hétérogènes. Alors que, la date de $50 \%$ de floraison (CV=3,700 \%), la durée du cycle (CV=7,40\%), le rendement en gousses par plante $(\mathrm{CV}=10,05 \%)$, le rendement en graines par plante $(\mathrm{CV}=14,87 \%)$ et la teneur en huile $(27,50 \%)$, montrent que ces 20 accessions sont homogènes. L'homogénéité de l'échantillon peut être due à l'influence des conditions environnementales (facteurs climatiques et édaphiques) sur ces cinq caractères. L’hétérogénéité de l'échantillon pour les quatre caractères montre que, ces caractères seraient moins influencés par les conditions environnementales. 
En effet, le taux de germination (TAG) varie entre 22, $66 \%$ et 100 $\%$ selon les accessions. Ce taux de germination est l'un des critères de la certification des semences de l'arachide. Il renvoie à la capacité d'une semence de réaliser toutes les fonctions essentielles pour la croissance normale d'une plante dans des conditions idéales (température, humidité et aération convenables à sa germination). Le résultat, montre que le taux de germination devient de plus en plus faible au fur et à mesure que la durée de la conservation devient importante. Mais, cela n'est pas valable pour les accessions conservées pendant 4 ans et 5 ans. Le faible taux de germination pourrait être dû soit à la longue durée de conservation, soit à la dormance favorisant la pourriture des graines. Selon Singh et al., (1991), les graines de nombreuses espèces d'Acacia utilisées en Inde ne germent pas lorsque le potentiel hydrique atteint -9 ou -12 bars. Selon Neffati et al., (1997) et Wahbi et al., (2010), les légumineuses des zones arides sont capables de germer sous une large gamme de températures $\left(15^{\circ} \mathrm{C}\right.$ et $\left.40^{\circ} \mathrm{C}\right)$. Ceci montre que la température n'est pas le facteur affectant du taux de germination des accessions conservées pendant 4 ans. Puisque, la température durant l'expérimentation est comprise entre $27,3^{\circ} \mathrm{C}$ et $30,7^{\circ} \mathrm{C}$. Il en est de même pour l'eau, car selon Ndour et al., (1998), l'eau n'est pas un facteur limitant de la germination chez les légumineuses de zones arides.

La surface foliaire des accessions varie entre 13,95 $\mathrm{cm}^{2}$ (A14) et $34,73 \mathrm{~cm}^{2}$ (A17). Cette surface foliaire des types taxonomiques HYB $(17,58$ $\left.\mathrm{cm}^{2}\right)$ et HYR $\left(16,80 \mathrm{~cm}^{2}\right)$ est plus étroite que celle des FST $\left(26,06 \mathrm{~cm}^{2}\right)$, PRU $\left(24,20 \mathrm{~cm}^{2}\right)$ et VUL $\left(26,84 \mathrm{~cm}^{2}\right)$. Cette étroite surface foliaire observée chez les types HYR et HYB pourrait être une stratégie d'augmentation de leur nombre des feuilles afin de recouvrir toute la surface du sol, donc une stratégie d'avoir un LAI.

L'indice de surface foliaire (LAI) varie entre 0,59 (A15) et 4,33 (A7). Ceci montre que le caractère rampant ne conditionne pas le recouvrement du sol par la plante. Les types taxonomiques PRU $(1,25)$, FST $(1,63)$ et VUL $(1,67)$ ont un LAI plus grand que les types taxonomiques HYB $(1,88)$ et HYR $(2,26)$. Cette importante valeur de LAI chez les HYB et HYR, confirme le caractère rampant de la sous espèce Arachis hypogaea hypogaea.

La hauteur de la tige des plantes varie entre $7 \mathrm{~cm}$ (A15) et 24,16 cm (A13). Elle est inferieure à celle de RABECHAULT (1960). Elle varie selon les types taxonomiques. Elle est plus petite chez les types taxonomiques HYB $(8,95)$ et HYR $(9,26 \mathrm{~cm})$ que chez les types VUL $(13,73 \mathrm{~cm})$, PRU $(16,69 \mathrm{~cm})$ et FST $(19,05 \mathrm{~cm})$. Ceci confirme aussi le caractère rampant de la sous espèce Arachis hypogaea hypogaea et les caractères érigé et semi érigé de la sous espèce Arachis hypogaea fastigiata. Elle est positivement et significativement corrélée à la surface foliaire $(\mathrm{r}=0,66)$. 


\begin{tabular}{|c|c|c|c|c|c|c|c|c|c|c|c|c|c|c|c|}
\hline Accessions & $50 \% \mathrm{~F}(\mathrm{j})$ & TAG (\%) & $\mathrm{SF}\left(\mathrm{cm}^{2}\right)$ & HPL (cm) & DC(j) & NGP & $\begin{array}{l}\text { RGP } \\
\text { (g/plante) }\end{array}$ & $\begin{array}{l}\text { RgP } \\
\text { (g/plante) }\end{array}$ & TEH (\%) & $\begin{array}{l}\text { DFL } \\
\text { (J) }\end{array}$ & \begin{tabular}{|l|}
$T$ \\
$(\%)$
\end{tabular} & LAI & NRG & Nmg/G & PMg \\
\hline A13 & $22,666^{\mathrm{a}}$ & 100,000 & $27,692^{c}$ & $24,167^{d}$ & $85,000^{c}$ & $33,267^{b}$ & $18,429^{\mathrm{a}}$ & $5,470^{a}$ & $51,300^{\mathrm{a}}$ & $\begin{array}{l}19 \\
\end{array}$ & 80 & 2,51 & 10 & 4 & 0,7 \\
\hline A19 & $23,000^{\mathrm{ab}}$ & 100,000 & $21,746^{\mathrm{c}}$ & $17,267^{d}$ & $93,333^{f}$ & $19,867^{\mathrm{a}}$ & $10,076^{\mathrm{a}}$ & $1,117^{\mathrm{a}}$ & $36,500^{\mathrm{a}}$ & 20 & 96,67 & 1,41 & 0 & 4 & 0,6 \\
\hline A20 & $23,000^{b}$ & 100,000 & $24,511^{\mathrm{c}}$ & $15,833^{d}$ & $90,000{ }^{\text {ef }}$ & $18,600^{\mathrm{a}}$ & $10,852^{\mathrm{a}}$ & $1,119^{\mathrm{a}}$ & $52,800^{\mathrm{a}}$ & 20 & 100 & 1,26 & 0 & 4 & 0,55 \\
\hline A6 & $24,000^{\text {bc }}$ & 96,667 & $30,181^{\mathrm{c}}$ & $15,333_{\mathrm{cd}}$ & $86,667^{\mathrm{cd}}$ & $42,667^{c}$ & $26,508^{c}$ & $18,572^{\mathrm{c}}$ & $36,800^{\mathrm{a}}$ & 23 & 76,67 & 1,58 & 12 & 2 & 0,44 \\
\hline A12 & $25,000^{\mathrm{cd}}$ & 63,333 & $24,487^{c}$ & $16,700^{d}$ & 88,333 de & $27,433^{b}$ & $11,970^{\mathrm{a}}$ & $1,877^{\mathrm{a}}$ & $43,800^{\mathrm{a}}$ & 21 & 56,67 & 1,29 & 14 & 4 & 0,43 \\
\hline A18 & $25,000^{d}$ & 70,000 & $19,037^{b}$ & $13,200^{\mathrm{c}}$ & $88,333^{\mathrm{e}}$ & $39,067^{c}$ & $18,510^{\mathrm{a}}$ & $7,870^{\mathrm{a}}$ & $49,300^{\mathrm{a}}$ & 22 & 70 & 1,08 & 10 & 3 & 0,44 \\
\hline A9 & 26,000 de & 70,000 & $26,850^{\mathrm{c}}$ & $13,767^{\mathrm{c}}$ & $85,000^{b c}$ & $56,633^{c}$ & $53,265^{c}$ & \begin{tabular}{|l}
$29,888^{c}$ \\
\end{tabular} & $41,500^{\mathrm{a}}$ & 23 & 70 & 2,22 & 12 & 2 & 0,65 \\
\hline A16 & 26,000 e & 73,333 & $17,867^{b}$ & $10,500^{b}$ & $91,667 \quad f$ & $55,100^{c}$ & $30,943^{c}$ & $12,977^{\text {bc }}$ & $41,400^{\mathrm{a}}$ & 24 & 73,33 & 2,8 & 12 & 3 & 0,52 \\
\hline A11 & 26,000 e & 46,667 & $22,808^{\mathrm{c}}$ & $21,300^{d}$ & $\begin{array}{|ll|}91,667 \quad f \\
\end{array}$ & $35,367^{b}$ & $15,592^{\mathrm{a}}$ & $9,612^{\mathrm{ab}}$ & $49,200^{\mathrm{a}}$ & 22 & \begin{tabular}{|l|}
46,67 \\
\end{tabular} & 1,07 & 0 & 3 & 0,56 \\
\hline A10 & 26,000 e & 76,333 & $29,181^{\mathrm{C}}$ & $13,267^{\mathrm{c}}$ & $81,667^{\mathrm{ab}}$ & $33,967^{b}$ & $22,265^{\mathrm{ab}}$ & $14,114^{\mathrm{c}}$ & $50,900^{\mathrm{a}}$ & 23 & 76,67 & 1,2 & 12 & 2 & 0,42 \\
\hline A8 & 26,000 & $96,667 \quad d$ & $20,984^{c}$ & $12,560^{\mathrm{bc}}$ & $80,000^{\mathrm{a}}$ & $30,567^{b}$ & $16,549^{\mathrm{a}}$ & $5,970^{\mathrm{a}}$ & $36,300^{\mathrm{a}}$ & 24 & 96,67 & 1,68 & 11 & 2 & 0,47 \\
\hline A1 & 27,000 & $30,000^{b}$ & $26,080^{c}$ & $16,967^{d}$ & 98,333 & $32,167^{\mathrm{b}}$ & $14,883^{\mathrm{a}}$ & $1,295^{\mathrm{a}}$ & $42,300^{\mathrm{a}}$ & 33,333 & 24 & 1,06 & 10 & 3 & 0,4 \\
\hline A7 & 27,000 & $63,333 \quad d$ & $14,303^{b}$ & $11,330^{b}$ & 96,667 & $71,333^{c}$ & $57,749^{\mathrm{c}}$ & $30,834^{\mathrm{c}}$ & $51,600^{\mathrm{a}}$ & 24 & 46,67 & 4,33 & 11 & 3 & 0,62 \\
\hline A4 & 27,000 & $36,667^{\mathrm{b}}$ & $18,349^{b}$ & $10,330^{b}$ & 95,000 & $43,100^{c}$ & $38,783^{c}$ & $14,234^{\mathrm{c}}$ & $51,100^{\mathrm{a}}$ & 24 & 33,33 & 2,26 & 12 & 3 & 0,91 \\
\hline A3 & 28,000 & $50,000 \quad d$ & $20,086^{\mathrm{bc}}$ & $8,500^{\mathrm{b}}$ & 93,333 & $30,200^{b}$ & $9,209^{\mathrm{a}}$ & \begin{tabular}{|l|}
$1,774^{\mathrm{a}}$ \\
\end{tabular} & $39,500^{\mathrm{a}}$ & 24 & \begin{tabular}{|l|}
46,67 \\
\end{tabular} & 2,23 & 12 & 2 & 0,55 \\
\hline A17 & 29,000 & $60,000 \quad d$ & $34,731^{c}$ & $17,567^{d}$ & \begin{tabular}{|ll}
88,333 & e \\
\end{tabular} & $23,833^{\mathrm{ab}}$ & $15,252^{\mathrm{a}}$ & $4,233^{\mathrm{a}}$ & $44,300^{\mathrm{a}}$ & 24 & 60 & 1,87 & 12 & 4 & 0,71 \\
\hline A2 & 29,000 & $30,000^{a b}$ & $17,938^{\mathrm{b}}$ & $9,400^{b}$ & 95,000 & $43,333^{c}$ & $25,078^{\text {bc }}$ & $5,742^{\mathrm{a}}$ & $52,800^{\mathrm{a}}$ & 23 & \begin{tabular}{|l|}
30 \\
\end{tabular} & 1,95 & 12 & 2 & 0,55 \\
\hline A14 & 29,333 & $43,333^{\mathrm{bc}}$ & $13,951^{\mathrm{a}}$ & $7,867^{\mathrm{ab}}$ & 95,000 & $38,967^{\text {bc }}$ & $14,210^{\mathrm{a}}$ & $4,364^{\mathrm{a}}$ & $37,600^{\mathrm{a}}$ & 23 & 43,33 & 1,08 & 12 & 2 & 0,48 \\
\hline A5 & 30,000 & $56,667 \quad d$ & $20,948^{c}$ & $8,733^{b}$ & 95,000 & $24,600^{b}$ & $11,970^{\mathrm{a}}$ & $5,257^{\mathrm{a}}$ & 46,1 & 25 & 56,67 & 1,34 & 12 & 2 & 0,53 \\
\hline A15 & 35,000 & $26,667^{\text {a }}$ & $14,119^{\mathrm{ab}}$ & $7,000^{a}$ & 96,667 & $33,100^{b}$ & $10,089^{\mathrm{a}}$ & $4,353^{\mathrm{a}}$ & 43,3 & 20 & 26,67 & 0,59 & 10 & 2 & 0,31 \\
\hline Moyenne & 26,7 & 64,833 & 22,302 & 13,555 & 90,75 & \begin{tabular}{|l|}
36,658 \\
\end{tabular} & 21,61 & 9,034 & 44,92 & 23,067 & 60,53 & 1,7405 & 9,8 & 2,8 & 0,542 \\
\hline CV(\%) & 3,7 & 44,2 & 35,9 & 44,7 & 7,4 & 57,8 & 10,05 & 14,87 & 27,5 & 1,128 & 3,88 & 4,82 & $4 ? 442$ & 2,98 & 2,48 \\
\hline ppds /HSD & \begin{tabular}{|l|}
2,021 \\
\end{tabular} & 3,728 & 2,021 & 2,453 & 2,021 & 2,021 & 2,021 & 2,021 & 2,086 & - & - & - & -- & - & - \\
\hline Ecart type & 3,233 & 28,671 & 8,011 & 6,057 & 6,691 & 21,171 & 21,61 & 13,433 & 12,343 & 2,953 & 23,47 & 0,8384 & 4,3359 & 0,834 & 0,134188 \\
\hline Ficher & $7,079 * * *$ & $6,041 * * *$ & $2,005 *$ & $2,657 * *$ & $2,950 * *$ & $1,206^{n s}$ & $0,119^{\mathrm{ns}}$ & $1,496^{\mathrm{ns}}$ & $0,296^{\text {ns }}$ & - & - & - & - & - & - \\
\hline
\end{tabular}

Tableau 4: Performance de chaque accession d'arachide pour les 15 caractères quantitatifs

***, très hautement significative;**, très significative;* significative; 50 \% F, cinquante pourcent de floraison; DC, durée du cycle; DFL, Début de floraison; HPL, hauteur de la plante; ICG, Icrisat Centre Groundunt; LAI, indice de surface foliair ; NGP, nombre de gousses par plante; PMg, Nmg/G, nombre maximum de graines par gousse; NRG, nombre de réticulations par gousse; poids moyenne d’une graine; RGP, rendement en gousses par plante; SF, surface foliaire; TAG, taux de germination. Les moyennes suivies par les mêmes lettres ne sont pas significativement différentes à 95 \% de niveau de confiance 
La matrice de corrélation a été établie afin de savoir, si la teneur en huile est corrélée à d’autres caractères (Tableau 5).

Tableau 5: Matrice de corrélation

\begin{tabular}{|c|c|c|c|c|c|c|c|c|c|c|c|c|c|c|c|}
\hline & TAG & $\mathbf{T}$ & DFL & $50 \%$ F & SF & HPL & LAI & DC & NGP & NRG & Nmg/G & RGP & RgP & PMg 1 & TEH \\
\hline TAG & 1 & & & & & & & & & & & & & & \\
\hline $\mathbf{T}$ & $0,961 * * *$ & & 1 & & & & & & & & & & & & \\
\hline DFL & $-0,430$ & $-0,409$ & 1 & & & & & & & & & & & & \\
\hline $50 \% \mathrm{~F}$ & $-0,776 * *$ & $-0,715$ & $* * 0,324$ & 1 & & & & & & & & & & & \\
\hline SF & 0,424 & 0,398 & $-0,040$ & $-0,427$ & 1 & & & & & & & & & & \\
\hline HPL & 0,481 & 0,405 & $-0,471$ & $-0,674 *$ & 0,668* & 1 & & & & & & & & & \\
\hline LAI & 0,140 & 0,007 & 0,310 & $-0,183$ & $-0,150$ & $-0,049$ & 1 & & & & & & & & \\
\hline DC & $-0,680 *$ & $-0,704$ & $* 0,262$ & 0,489 & $-0,572 *$ & $-0,393$ & 0,054 & 1 & & & & & & & \\
\hline NGP & $-0,172$ & $-0,272$ & 0,390 & 0,060 & -0,354 & $-0,272$ & $0,701 *$ & 0,117 & 1 & & & & & & \\
\hline NRG & $-0,299$ & $-0,362$ & $0,513^{*}$ & 0,371 & $-0,016$ & $-0,446$ & 0,257 & $-0,102$ & 0,378 & 1 & & & & & \\
\hline Nmg/G & 0,346 & 0,310 & $-0,445$ & $-0,521 *$ & 0,310 & $0,677^{*}$ & 0,107 & 0,107 & $-0,312$ & $-0,434$ & 1 & & & & \\
\hline RGP & $-0,050$ & $-0,138$ & 0,339 & $-0,031$ & $-0,151$ & $-0,171$ & 0,7204 & 0,004 & 0,901 & 0,301 & $-0,188$ & 1 & & & \\
\hline RgP & $-0,050$ & $-0,009$ & 0,341 & $-0,090$ & $-0,022$ & $-0,105$ & $0,619 *$ & $0,619 *$ & $0,856 * *$ & 0,254 & $-0,287$ & $0,939 * * *$ & 1 & & \\
\hline PMg & 0,057 & 0,017 & 0,156 & $-0,244$ & 0,124 & 0,196 & $0,537 *$ & 0,025 & 0,163 & $-0,054$ & 0,356 & 0,396 & 0,261 & 1 & \\
\hline
\end{tabular}

*, corrélation faible;** corrélation forte, ***, corrélation très forte; $(+)$ et $(-)$ corrélation significativement :positivement et négativement corrélés respectivement; chiffre en gras montre une corrélation significative; 50 \% F, cinquante pour cent de floraison; DC, durée du cycle; DFL, Début de floraison; HPL, hauteur de la plante; NGP, nombre de gousses par plante; Nmg/G, nombre maximum de graines par gousse; NRA, nombre des réticulation par gousse; PMg, poids moyenne d'une graine; RGP , rendement en gousses par plante; RgP, rendement en graine par plante; SF, surface foliaire; T, taux de levée; TAG, taux de germination; TEH, teneur en huile.

Afin de décrire les types taxonomiques des accessions, une Analyse Factorielle Discriminante (AFD) a été faite sur la base des quinze caractères retenus lors des observations. En effet, excepté le taux de germination, 14 caractères permettant de discriminer les différents types taxonomiques ont été retenus. Ces types taxonomiques sont statistiquement différents (Lambda $=0,0000001462$, F critique $(2,645)<$ observé $(10,02)$ au risque 0,05 avec un taux d'erreur d'apparence= 0,0005 ). Le type taxonomique fastigiata fastigiata Valencia (FST) est le type taxonomique le plus riche en huile, que les autres types taxonomiques (PRU, VUL, HYR et HYB). Les carrés de distance de Mahalanobis issus de l'AFD montrent que le type taxonomique VUL $(2129,25)$ est plus proche de celui de FST que les types HYR, HYB et PRU. Les types HYR, FST et HYB sont plus proches de VUL, que PRU. Par contre, le type FST est plus loin de HYB et HYR que VUL et PRU (Tableau 6). Chaque caractère est spécifique au type taxonomique. Les 14 caractères permettant de discriminer les types taxonomiques sont présentés dans le Tableau 7. 
Tableau 6: Carrés de distance de Mahalanobis entre les types taxonomiques

\begin{tabular}{|l|l|l|l|l|l|}
\hline & FST & HYB & HYR & PRU & VUL \\
\hline FST & 0 & & & & \\
\hline HYB & 7618,316 & 0 & & & \\
\hline HYR & 2270,586 & 1622,521 & 0 & & \\
\hline PRU & 3294,927 & 20761,289 & 10911,307 & 0 & \\
\hline VUL & 2129,258 & 17426,535 & 8579,116 & 452,924 & 0 \\
\hline
\end{tabular}

FST, fastigiata fastigiata Valancia; HYB, hypogaea bunch Virginia bunch; HYR, hypogaea runner Virginia runner; PRU, fastigiata peruviana Valencia peruviana; VUL, fastigiata vulgaris Spanish.

Tableau 7: Performance moyennes des groupes taxonomiques

\begin{tabular}{|l|l|l|l|l|l|l|l|l|l|}
\hline Groupes & FST & HYB & HYR & PRU & \multicolumn{1}{|l|}{ VUL } & \multicolumn{1}{|l|}{$\begin{array}{l}\text { Ecart } \\
\text { type }\end{array}$} & CV(\%) & Fisher \\
\hline Fréquence & 4 & 4 & 4 & 4 & 4 & Moyennes \\
\hline T (\%) & 64,167 & 38,333 & 50,833 & 70,833 & 80,000 & 60,833 & 16,468 & 27,1 & $2,551^{\text {ns }}$ \\
\hline DFL (J) & 21,750 & 23,500 & 23,250 & 21,250 & 22,750 & 22,5 & 0,968 & 4,3 & $2,167^{\text {ns }}$ \\
\hline 50\% F & 25,667 & 28,333 & 29,500 & 24,500 & 25,500 & 26,7 & 2,113 & 7,9 & $426,449^{*}$ \\
\hline SF cm2) & 26,067 & 17,581 & 16,809 & 24,206 & 26,849 & 22,302 & 4,465 & 21,4 & $98,803^{*}$ \\
\hline HPL (cm) & 19,059 & 8,950 & 9,267 & 16,692 & 13,734 & 13,54 & 4,465 & 33 & $1,543^{\text {ns }}$ \\
\hline LAI & 1,633 & 1,880 & 2,265 & 1,255 & 1,670 & 1,741 & 0,37 & 21,2 & $265,942^{*}$ \\
\hline DC (J) & 88,333 & 94,583 & 95,000 & 92,500 & 83,334 & 90,7 & 4,916 & 5,4 & $2,245^{\text {ns }}$ \\
\hline NGP & 32,884 & 38,900 & 46,033 & 24,517 & 40,959 & 36,659 & 8,262 & 22,5 & $2,458^{\text {ns }}$ \\
\hline NRG(g) & 8,000 & 12,000 & 11,250 & 6,000 & 11,750 & 9,8 & 2,666 & 27,2 & $1,014^{\text {ns }}$ \\
\hline Nmg/G & 3,500 & 2,250 & 2,500 & 3,750 & 2,000 & 2,8 & 0,779 & 27,8 & $0,385^{\text {ns }}$ \\
\hline RGP (g) & 16,946 & 21,820 & 29,188 & 11,945 & 29,647 & 21,909 & 7,694 & 35,1 & $0,645^{\text {ns }}$ \\
\hline RgP (g) & 6,797 & 6,529 & 13,355 & 1,352 & 17,136 & 9,034 & 6,217 & 68,8 & $0,631^{\text {ns }}$ \\
\hline PMg (g) & 0,603 & 0,623 & 0,495 & 0,495 & 0,495 & 0,542 & 0,065 & 12 & $0,633^{\text {ns }}$ \\
\hline TEH (\%) & 48,525 & 45,250 & 45,600 & 43,850 & 41,375 & 44,92 & 2,611 & 5,8 & $0,022^{\text {ns }}$ \\
\hline
\end{tabular}

*, significative; ${ }^{\mathbf{n}}$, non significative; DC, durée du cycle; DFL, début de floraison; F, fréquence; FST, fastigiata fastigiata Valancia; HPL, hauteur de la plante; HYB, hypogaea bunch Virginia bunch; HYR, hypogaea runner Virginia runner LAI, indice de surface foliaire; NGP, nombre des gousses par plante; Nmg/G, nombre maximum de graines par gousse ;PMg, poids moyen d'une graine; PRU, fastigiata peruviana Valencia peruviana; SF, surface foliaire; RGP, rendement en gousses par plante; RgP, rendement en graines par plante; TEH, teneur en huile VUL, fastigiata vulgaris Spanish.

\section{Conclusion}

La caractérisation a relevé, une certaine hétérogénéité pour les caractères tels que la surface foliaire, la hauteur de la plante, le nombre de gousses par plante et le taux de germination. Tous les caractères mesurés, à l'exception du taux germination, permettent de discriminer les types taxonomiques. Le type taxonomique fastigiata fastigiata Valencia est le meilleur taxon riche en huile. 
Quant aux accessions, les accessions A20 $(52,8)$ et A2 $(52,8)$ sont les meilleures accessions en matière de richesse en huile. La richesse en huile d'arachide est liée au poids moyen d'une graine. Les accessions qui ont un très grand poids moyen d'une graine et de type taxonomique fastigiata fastigiata Valencia pourraient être recommandées comme un critère de sélection pour identifier les arachides d'industries d'huilerie. Une étude, pourrait être réalisée uniquement au sein du type taxonomique fastigiata fastigiata Valencia afin de savoir si la valeur de la corrélation entre la teneur en huile et le poids d'une graine sera supérieure à 0,312\%. Il est aussi recommandé d'approfondir les études sur la composition chimique de l'accession A17 afin de savoir la cause exacte du changement de la couleur de son huile.

\section{References:}

Bouffil F., 1951. Biologie évolution et sélection de l'arachide au Sénégal. Cell Tech. Agric. Trop., 111p.

Celia N. et Synthia S., 2005. L'allergie alimentaire à l'arachide, Univ. Paris XII-vol. de Marne 61. Avenue du General de Gaulle 94010 Creteil Cedex, 40p.

Conkertone e. E., J. et ory L., 1989. Les effets du stress hydrique sur la composition d'arachide, vol.1, 144 (12), pp. 593-602.

Gregory W. C. and Gregory M. P., 1967. Indice mutation and species hybridization and depreciation of arachis. Ciencia culture; 19, pp. 166-174.

Hammons S., 1973. The origin and history of the groundnut Landers Royaume. Crop, J., Smartt, Uni., chapman and Hall, pp. 24-42.

Herselman L., 2003. Genetic variation among Southern African cultivated peanut (Arachis hypogaea L.) genotypes as revealed by AFLP analysis. Euphytica; 133, pp. 27-319.

IBPGR (Now Biodiversity international) and ICRISAT, 1992. Descriptors for groundnut, 125p.

Khalfaoui J. L., 1988. Approche de l'amélioration génétique de l'adaptation à la sécheresse des espèces cultivées en zone semi- arides. Application au cas de l'arachide (Arachis hypogaea L.) destinée à la région sèche du Sénégal. Thes. Doct.Univ. du Paris-Sud., pp. 67-68.

Kravickas A, 1969. The origin variability and spread of the groundnut (Arachis hypgaea L.) In :The Domestication and exploitation of plant and animals Ucko R.J. Dimbledy W. C. (Eds.). Londres GB : Duckworth, pp. 40427.

Kumaga F. K., Adiku S .G. K. and Ofori K., 2003. Effect of post-floering Water stress on dry matter and yield of three Tropical Grain legumes. Int. J. Agric. Biol., 4 pp. 405-407. 
Makanda I. Tongoona P., Maamba R., Icishahayo D. and Derera J., 2009. Path coefficient analysis of bambara groundnut pod yield components at for planting dates. Res. J. Agric. Biol. Sc., 5 (3), pp. 289-292.

Mayeux A. H., 1999. Etude sur le taux d'allogamie chez l'arachide. Oléagineux, 18, pp. 571-574.

Ndour P. et Danthu P., 1998. Effet des contraintes hydriques et amines sur la germination de quelques Acacia africana. Campa C., Grignon C., Gueye M. et Hamon S., eds., colloque et séminaires: l'acacia au Sénégal. Paris: ORSTOM, pp. 105-122.

Neffati M., 1997. Caractérisation morpho-biologique de certaines espèces végétales nord africaines: implication pour l'amélioration pastorale. Thès. Doc. Univ. Grande Bretagne (Belgique), Sp.

Nigam S. N. Rao V. Ramanatha and Gibbons R. W., 1983. Utilization of natural hybrids in the improvement of groundnut (Arachis hypogaea L.). Exp. Agric., 19, pp. 355-359.

Onwubiko N. I.C., Odum O. B. Uttazi C.O. and Poly- Mbah P. C., 2011. Studies on the adaptation of bambara groundnut (Vigna subterranea L. Verdc): In owerri Southeastern Nigeria. New York Sc. J., 4(2), pp. 60-67.

Rabechault H., 1960. Recueil iconographique des espèces comestibles de légumineuses africaines. « Riz et riziculture » Paris, 61p.

Sauger L., 1949. Hybridation de l'arachide à Bambey. Paris. Agr. Trop., vol. 4,12, pp. 618-624.

Singh A., Smaartt J., Simpson C. E. and Raina S. N., 1998. Genetic variation molecular polymorphism in groundnut (Arachis hypogaea L.). Gen. Res.Crop Eval., 45, pp. 26-119.

Singh C., Khajuria H. N. Singh A. and Sharma F., 1991. Acacias forarid regions. Acta. Bot. indica, 19, pp. 29-32.

Wahbi jaouad, Lamia H., Naoufel S. et Mohamed L. K., 2010. Etude de la germination des graines d'Acacia tortilis sous différentes contraintes abiotiques. Biotechnol. Agron. Soc. Environ.14(4), pp. 643-652. 